\title{
A system for near real-time detection of filament eruptions at Kanzelhöhe Observatory
}

\author{
Werner Pötzi ${ }^{1}$, Gernot Riegler ${ }^{2}$, Astrid Veronig ${ }^{1}$, Thomas Pock $^{2}$ and \\ Ute Möstl ${ }^{1}$ \\ ${ }^{1}$ University of Graz, Institue of Physics, IGAM-Kanzelhöhe Observatory, Austria \\ ${ }^{2}$ Technical university of Graz, Institue for Computer Graphics and Vision, Austria
}

\begin{abstract}
Kanzelhöhe Observatory (kso.ac.at) performs regular high-cadence full-disk observations of the solar chromosphere in the $\mathrm{H} \alpha$ and CaIIK spectral lines as well as the solar photosphere in white-light. In the frame of ESA's Space Situational Awareness (SSA) activities, a new system for near real-time $\mathrm{H} \alpha$ image provision through the SSA Space Weather (SWE) portal (swe.ssa.esa.int) and for automatic alerting of flares and erupting filaments is under development. Image segmentation algorithms, based on optical flow image registration, for the automatic detection of solar filaments in real time $\mathrm{H} \alpha$ images have been developed and implemented at the Kanzelhöhe observing system. We present first results of this system with respect to the automatic recognition and segmentation of filaments and filament eruptions on the Sun.
\end{abstract}

Keywords. $\mathrm{H} \alpha$, filaments, solar activity, image processing

\section{Introduction and Data}

Full disc $\mathrm{H} \alpha$ images provided by the $\mathrm{H} \alpha$ telescope at Kanzelhöhe Observatory with a focal length of $2000 \mathrm{~mm}$ and an aperture of $100 \mathrm{~mm}$ are used for our purpose. The telescope is equipped with a Lyot filter centered at $656.28 \mathrm{~nm}$ and a FWHM of $0.07 \mathrm{~nm}$. The time cadence of the 4 Megapixel images (resolution $1 \mathrm{arcsec} / \mathrm{pixel}$ ) is 6 seconds and the system makes use of the frame selection technique, i.e. the camera software grabs the best image based on the image contrast during a preselected time interval. A fast computer is connected to the real-time observations, which analyses the incoming $\mathrm{H}$ alpha images with respect to filaments and flares, and sends the output in near real-time to the SSA SWE portal (http://swe.ssa.esa.int).

\section{The KSO SSA SWE subportal \& Filament Eruption recognition}

On the ESA SWE portal the observatory hosts a subportal (Fig. 1) displaying near real-time $\mathrm{H} \alpha$ images and movies and lists of flares and filament eruptions. Every minute the page is refreshed and a new $\mathrm{H} \alpha$ image is displayed. Detected flares and filament eruptions are shown in a table. Additionally a 360 degree view of a web-cam informs about the actual weather conditions at the observatory.

Filaments are detected by a highly parallelized feature recognition algorithm (Riegler et al. (2013)). The system is able to process up to 8 images per minute. Each feature is labeled and tracked during the whole observing day. For each filment, its ID, position and length are saved into log-files for further processing. 


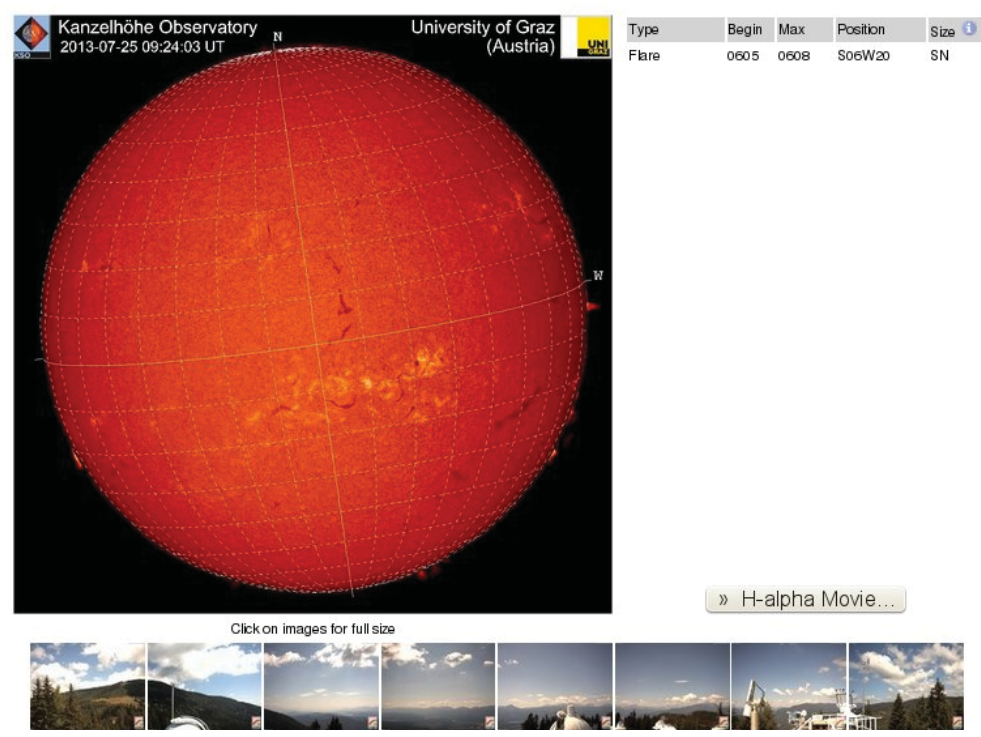

Figure 1. Sub portal design of the Kanzelhöhe $\mathrm{H} \alpha$ services on ESA's SSA SWE portal. With a click on the image a new window pops up showing the $\mathrm{H} \alpha$ image at $1024 \times 1024$ pixels and a daily history. The button "H-alpha movie" shows an interface, where a movie of the latest hour of observations is visible including the posiblity to control the movie speed and direction. Flares and filaments automatically detected in near real-time are displayed in a table. The interface is updated every minute.

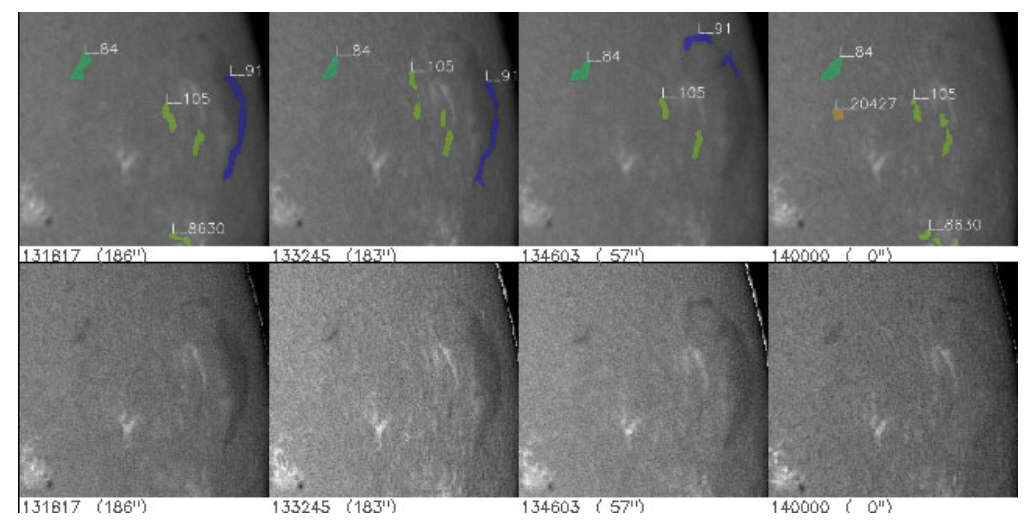

Figure 2. Bottom: sequence of KSO Halpha images, top: same sequence but with the output of the automated filament recognition overplotted. The filament close to the limb disappears from the $3^{r d}$ to the $4^{\text {th }}$ image. After 14:00 the filament was not detected anymore by the algorithm and it was therefore identified as filament eruption.

If a filament ID disappears for a certain time, a filament eruption alert is provided on the SWE H-alpha subportal.

\section{Acknowledgements}

This study was developed within the framework of ESA's SSA programme (SWE SN IV-2 activity).

\section{Reference}

Riegler, G., Pock, T., Pötzi, W., \& Veronig A. 2013, arXiv:1304.7132 